\title{
Nemat Nyamoradi · Mohsen Shekarbigi \\ Existence of positive solutions for a quasilinear elliptic system involving critical Sobolev-Hardy exponents and concave-convex nonlinearities
}

Received: 27 August 2012 / Accepted: 8 July 2013 / Published online: 2 August 2013

(C) The Author(s) 2013. This article is published with open access at Springerlink.com

\begin{abstract}
This paper is concerned with a quasilinear elliptic system, which involves the Caffarelli-KohnNirenberg inequality and multiple critical exponents. The existence and multiplicity results of positive solutions are obtained by variational methods.
\end{abstract}

Mathematics Subject Classification $35 \mathrm{~A} 15 \cdot 35 \mathrm{~B} 33 \cdot 35 \mathrm{~J} 70$

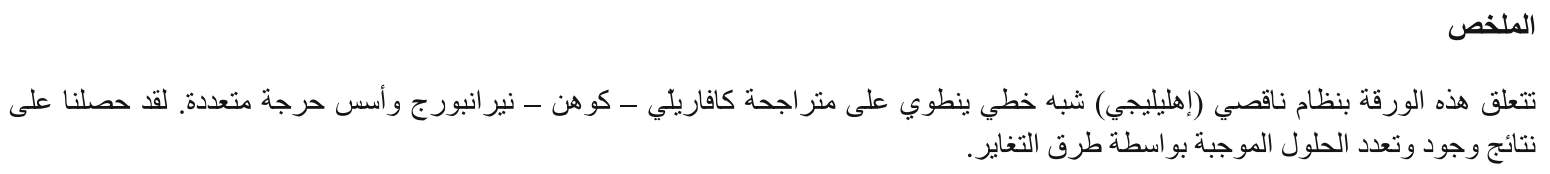

\section{Introduction}

The aim of this paper is to establish the existence of nontrivial solutions to the following quasilinear elliptic system

$$
\begin{cases}-\Delta_{p} u-\mu \frac{|u|^{p-2} u}{|x|^{p}}=\frac{p \alpha}{\alpha+\beta} \frac{|u|^{\alpha-2}|v|^{\beta} u}{|x|^{t}}+\lambda \frac{|u|^{q-2} u}{|x|^{s}}, & x \in \Omega, \\ -\Delta_{p} v-\mu \frac{|v|^{p-2} v}{|x|^{p}}=\frac{p \beta}{\alpha+\beta} \frac{|u|^{\alpha}|v|^{\beta-2} v}{|x|^{t}}+\theta \frac{|v|^{q-2} v}{|x|^{s}}, & x \in \Omega, \\ u=v=0, & x \in \partial \Omega\end{cases}
$$

where $\Delta_{p} u=\operatorname{div}\left(|\nabla u|^{p-2} \nabla u\right), 0 \in \Omega$ is a bounded domain in $\mathbb{R}^{N}(N \geq 3)$ with smooth boundary $\partial \Omega$, $\lambda>0, \theta>0,1<p<N, 0 \leq \mu<\bar{\mu} \triangleq\left(\frac{N-p}{p}\right)^{p}, 0 \leq s, t<p, 1 \leq q<p, \alpha+\beta=p^{*}(t) \triangleq \frac{p(N-t)}{N-p}$ is the Hardy- Sobolev critical exponent.

We denote by $D_{0}^{1, p}(\Omega)$ the completion of $C_{0}^{\infty}(\Omega)$ with respect to the norm $\left(\int_{\Omega}|\nabla \cdot|^{p} d x\right)^{\frac{1}{p}}$.

Problem (1) is related to the well known Caffarelli-Kohn-Nirenberg inequality in [13]:

$$
\left(\int_{\Omega} \frac{|u|^{r}}{|x|^{t}} d x\right)^{\frac{p}{r}} \leq C_{r, t, p} \int_{\Omega}|\nabla u|^{p} d x, \quad \text { for all } u \in D_{0}^{1, p}(\Omega),
$$

where $p \leq r<p^{*}(t)$. If $t=r=p$, the above inequality becomes the well known Hardy inequality $[13,18,19]:$

N. Nyamoradi $(\bowtie) \cdot$ M. Shekarbigi

Department of Mathematics, Faculty of Science,

Razi University, 67149 Kermanshah, Iran

E-mail: neamat80@yahoo.com; nyamoradi@razi.ac.ir 


$$
\int_{\Omega} \frac{|u|^{p}}{|x|^{p}} d x \leq \frac{1}{\bar{\mu}} \int_{\Omega}|\nabla u|^{p} d x, \text { for all } u \in D_{0}^{1, p}(\Omega) .
$$

In the space $D_{0}^{1, p}(\Omega)$ we employ the following norm:

$$
\|u\|=\|\left. u\right|_{D_{0}^{1, p}(\Omega)}:=\left(\int_{\Omega}\left(|\nabla u|^{p}-\mu \frac{|u|^{p}}{|x|^{p}}\right) d x\right)^{\frac{1}{p}}, \quad \mu \in[0, \bar{\mu}) .
$$

Using the Hardy inequality (3), this norm is equivalent to the usual norm $\left(\int_{\Omega}|\nabla u|^{p} d x\right)^{\frac{1}{p}}$. The elliptic operator $L:=\left(|\nabla \cdot|^{p-2} \nabla \cdot-\mu \frac{|\cdot|^{p-2}}{|x|^{p}}\right)$ is positive in $D^{1, p}(\Omega)$ if $0 \leq \mu<\bar{\mu}$.

Now, we define the space $W=D_{0}^{1, p}(\Omega) \times D_{0}^{1, p}(\Omega)$ with the norm

$$
\|(u, v)\|^{p}=\|u\|^{p}+\|v\|^{p} .
$$

Also, by Hardy inequality and Hardy-Sobolev inequality, for $0 \leq \mu<\bar{\mu}, 0 \leq t<p$ and $p \leq r \leq p^{*}(t)$ we can define the best Hardy-Sobolev constant:

$$
A_{\mu, t, r}(\Omega)=\inf _{u \in D_{0}^{1, p}(\Omega) \backslash\{0\}} \frac{\int_{\Omega}\left(|\nabla u|^{p}-\mu \frac{|u|^{p}}{|x|^{p}}\right) d x}{\left(\int_{\Omega} \frac{|u|^{r}}{|x|^{t}} d x\right)^{\frac{p}{r}}} .
$$

In the important case when $r=p^{*}(t)$, we simply denote $A_{\mu, t, p^{*}(t)}$ as $A_{\mu, t}$. Note that $A_{\mu, 0}$ is the best constant in the Sobolev inequality, namely,

$$
A_{\mu, 0}(\Omega)=\inf _{u \in D_{0}^{1, p}(\Omega) \backslash\{0\}} \frac{\int_{\Omega}\left(|\nabla u|^{p}-\mu \frac{|u|^{p}}{|x|^{p}}\right) d x}{\left(\int_{\Omega}|u|^{p^{*}} d x\right)^{\frac{p}{p^{*}}}} .
$$

For any $0 \leq \mu<\bar{\mu}, \alpha, \beta>1$ and $\alpha+\beta=p^{*}(t)$, by (2), (3), $0 \leq t<p$ we denote:

$$
\tilde{A}_{\mu, t}=\inf _{(u, v) \in W \backslash\{(0,0)\}} \frac{\int_{\Omega}\left(|\nabla u|^{p}+|\nabla v|^{p}-\mu \frac{|u|^{p}+|v|^{p}}{|x|^{p}}\right) d x}{\left(\int_{\Omega} \frac{|u|^{\alpha}|v|^{\beta}}{|x|^{t}} d x\right)^{\frac{p}{p^{*}(t)}}} .
$$

Modifying the proof of Theorem 5 in [2], we can easily deduce that

$$
\widetilde{A}_{\mu, t}=\left(\left(\frac{\alpha}{\beta}\right)^{\frac{\beta}{\alpha+\beta}}+\left(\frac{\alpha}{\beta}\right)^{\frac{-\alpha}{\alpha+\beta}}\right) A_{\mu, t} .
$$

Throughout this paper, let $R_{0}$ be the positive constant such that $\Omega \subset B\left(0 ; R_{0}\right)$, where $B\left(0 ; R_{0}\right)=\{x \in$ $\left.\mathbb{R}^{N}:|x|<R_{0}\right\}$. By Hölder and Sobolev-Hardy inequalities, for all $u \in D_{0}^{1, p}(\Omega)$, we obtain

$$
\begin{aligned}
\int_{\Omega} \frac{|u|^{q}}{|x|^{s}} & \leq\left(\int_{B\left(0 ; R_{0}\right)}|x|^{-s}\right)^{\frac{p^{*}(s)-q}{p^{*}(s)}}\left(\int_{\Omega} \frac{|u|^{p^{*}(s)}}{|x|^{s}}\right)^{\frac{q}{p^{*}(s)}} \\
& \leq\left(\int_{0}^{R_{0}} r^{N-s+1} d r\right)^{\frac{p^{*}(s)-q}{p^{*}(s)}} A_{\mu, s}^{-\frac{q}{p} \|\left. u\right|^{q}} \\
& \leq\left(\frac{N \omega_{N} R_{0}^{N-s}}{N-s}\right)^{\frac{p^{*}(s)-q}{p^{*}(s)}} A_{\mu, s}^{-\frac{q}{p}}\|u\|^{q},
\end{aligned}
$$

where $\omega_{N}=\frac{2 \pi^{\frac{N}{2}}}{N \Gamma\left(\frac{N}{2}\right)}$ is the volume of the unit ball in $\mathbb{R}^{N}$. 
Existence of nontrivial non-negative solutions for elliptic equations with singular potentials were recently studied by several authors, but, essentially, only with a solely critical exponent. We refer, e.g., in bounded domains and for $p=2$ to [14,19,20,27], and for general $p>1$ to $[15,16,21,22,25]$ and the references therein. For example, Kang in [25] studied the following elliptic equation via the generalized Mountain-Pass theorem [29]:

$$
\begin{cases}-\Delta_{p} u-\mu \frac{|u|^{p-2} u}{|x|^{p}}=\frac{|u|^{p^{*}(t)-2} u}{|x|^{t}}+\lambda \frac{|u|^{p-2} u}{|x|^{s}}, & x \in \Omega, \\ u=0, & x \in \partial \Omega\end{cases}
$$

where $\Omega \subset \mathbb{R}^{N}$ is a bounded domain, $1<p<N, 0 \leq s, t<p$ and $0 \leq \mu<\bar{\mu} \triangleq\left(\frac{N-p}{p}\right)^{p}$. Also, the authors in [17] via the Mountain-Pass Theorem of Ambrosetti and Rabinowitz [3], proved that

$$
-\Delta_{p} u-\mu \frac{u^{p-1}}{|x|^{p}}=|u|^{p^{*}-1}+\frac{u^{p^{*}(s)-1}}{|x|^{s}}, \quad \text { in } \mathbb{R}^{N}
$$

admits a positive solution in $\mathbb{R}^{N}$, whenever $\mu<\bar{\mu} \triangleq\left(\frac{N-p}{p}\right)^{p}$.

Also, in recent years, several authors have used the Nehari manifold and fibering method to solve semilinear and quasilinear problems (see [1,2,5,7-11,23] and references therein). Brown and Zhang [12] have studied a subcritical semi-linear elliptic equation with a sign-changing weight function and a bifurcation real parameter in the case $p=2$ and Dirichlet boundary conditions. In [24] the author studied the equation (7) via the Nehari manifold. Exploiting the relationship between the Nehari manifold and fibering maps (i.e., maps of the form $t \mapsto J_{\lambda}(t u)$ where $J_{\lambda}$ is the Euler function associated with the equation), they gave an interesting explanation of the well-known bifurcation result. In fact, the nature of the Nehari manifold changes as the parameter $\lambda$ crosses the bifurcation value.

In this work, we give a variational method which is similar to the fibering method (see $[7,12,24])$ to prove the existence and multiplicity of nontrivial non-negative solutions of Problem (1).

This paper consists of two sections along with an introduction. In Sect. 2, we establish some elementary results. In Sect. 3, we state our main results (Theorems 3.1, 3.3 and 3.7) and prove them.

\section{Notations and preliminaries}

The corresponding energy functional of Problem (1) is defined by

$$
J_{\lambda, \theta}(u, v)=\frac{1}{p}\|(u, v)\|^{p}-\frac{p}{\alpha+\beta} \int_{\Omega} \frac{|u|^{\alpha}|v|^{\beta}}{|x|^{t}} d x-\frac{1}{q} K_{\lambda, \theta}(u, v),
$$

for each $(u, v) \in W$, where $K_{\lambda, \theta}(u, v)=\lambda \int_{\Omega} \frac{|u|^{q}}{|x|^{s}} d x+\theta \int_{\Omega} \frac{|v|^{q}}{|x|^{s}} d x$. Then $J_{\lambda, \theta} \in C^{1}(W, \mathbb{R})$.

Now, we consider the problem on the Nehari manifold. Define the Nehari manifold (cf. [28]):

$$
N_{\lambda, \theta}=\left\{(u, v) \in W \backslash\{(0,0)\} \mid\left\langle J_{\lambda, \theta}^{\prime}(u, v),(u, v)\right\rangle=0\right\},
$$

where

$$
\left\langle J_{\lambda, \theta}^{\prime}(u, v),(u, v)\right\rangle=\|(u, v)\|^{p}-p \int_{\Omega} \frac{|u|^{\alpha}|v|^{\beta}}{|x|^{t}} d x-K_{\lambda, \theta}(u, v) .
$$

Note that $N_{\lambda, \theta}$ contains every nonzero solution of (1). Define

$$
\Phi_{\lambda, \theta}(u, v)=\left\langle J_{\lambda, \theta}^{\prime}(u, v),(u, v)\right\rangle
$$

then for $(u, v) \in N_{\lambda, \theta}$.

$$
\left\langle\Phi_{\lambda, \theta}^{\prime}(u, v),(u, v)\right\rangle=p\|(u, v)\|^{p}-p(\alpha+\beta) \int_{\Omega} \frac{|u|^{\alpha}|v|^{\beta}}{|x|^{t}} d x-q K_{\lambda, \theta}(u, v)
$$




$$
\begin{aligned}
& =(p-q)\|(u, v)\|^{p}-p\left(p^{*}(t)-q\right) \int_{\Omega} \frac{|u|^{\alpha}|v|^{\beta}}{|x|^{t}} d x \\
& =\left(p-p^{*}(t)\right)\|(u, v)\|^{p}-\left(q-p^{*}(t)\right) K_{\lambda, \theta}(u, v) .
\end{aligned}
$$

Now, we split $N_{\lambda, \theta}$ into three parts:

$$
\begin{aligned}
& N_{\lambda, \theta}^{+}=\left\{(u, v) \in N_{\lambda, \theta}:\left\langle\Phi_{\lambda, \theta}^{\prime}(u, v),(u, v)\right\rangle>0\right\}, \\
& N_{\lambda, \theta}^{0}=\left\{(u, v) \in N_{\lambda, \theta}:\left\langle\Phi_{\lambda, \theta}^{\prime}(u, v),(u, v)\right\rangle=0\right\}, \\
& N_{\lambda, \theta}^{-}=\left\{(u, v) \in N_{\lambda, \theta}:\left\langle\Phi_{\lambda, \theta}^{\prime}(u, v),(u, v)\right\rangle<0\right\} .
\end{aligned}
$$

To state our main result, we now present some important properties of $N_{\lambda, \theta}^{+}, N_{\lambda, \theta}^{0}$ and $N_{\lambda, \theta}^{-}$.

Lemma 2.1 There exists a positive number $C=C(p, q, N, S)>0$ such that if

$$
0<\lambda^{\frac{p}{p-q}}+\theta^{\frac{p}{p-q}}<C
$$

then $N_{\lambda, \theta}^{0}=\emptyset$.

Proof Suppose otherwise, let

$$
C=\left(\frac{p-q}{p\left(p^{*}(t)-q\right)}\right)^{\frac{p}{p^{*}(t)-p}}\left(\frac{p^{*}(t)-p}{p^{*}(t)-q}\right)^{\frac{p}{p-q}}\left(\frac{N \omega_{N} R_{0}^{N-s}}{N-s}\right)^{-\frac{p\left(p^{*}(s)-q\right)}{p^{*}(s)(p-q)}} A_{\mu, s}^{\frac{q}{p-q}} A_{\mu, t}^{\frac{p^{*}(t)}{p^{*}(t)-p}} .
$$

Then, there exists $(\lambda, \theta)$ with

$$
0<\lambda^{\frac{p}{p-q}}+\theta^{\frac{p}{p-q}}<C
$$

such that $N_{\lambda, \theta}^{0} \neq \emptyset$. Then for $(u, v) \in N_{\lambda, \theta}^{0}$, by (9) and (10) we have

$$
\|(u, v)\|^{p}=\frac{p\left(p^{*}(t)-q\right)}{p-q} \int_{\Omega} \frac{|u|^{\alpha}|v|^{\beta}}{|x|^{t}} d x .
$$

Moreover, by the Young inequality and (6), it follows that

$$
\begin{aligned}
\|(u, v)\|^{p} & =\frac{p\left(p^{*}(t)-q\right)}{p-q} \int_{\Omega} \frac{|u|^{\alpha}|v|^{\beta}}{|x|^{t}} d x \\
& \leq \frac{p\left(p^{*}(t)-q\right)}{p-q}\left(\frac{\alpha}{\alpha+\beta} \int_{\Omega} \frac{|u|^{\alpha+\beta}}{|x|^{t}} d x+\frac{\beta}{\alpha+\beta} \int_{\Omega} \frac{|v|^{\alpha+\beta}}{|x|^{t}} d x\right) \\
& \leq \frac{p\left(p^{*}(t)-q\right)}{p-q} A_{\mu, t}^{-\frac{p^{*}(t)}{p}}\|(u, v)\|^{\alpha+\beta} .
\end{aligned}
$$

It follows that

$$
\|(u, v)\| \geq\left(\frac{p-q}{p\left(p^{*}(t)-q\right)} A_{\mu, t}^{\frac{p^{*}(t)}{p}}\right)^{\frac{1}{p^{*}(t)-p}}
$$

and

$$
\begin{aligned}
\frac{p^{*}(t)-p}{p^{*}(t)-q}\|(u, v)\|^{p} & =K_{\lambda, \theta}(u, v)=\lambda \int_{\Omega} f \frac{|u|^{q}}{|x|^{s}} d x+\theta \int_{\Omega} g \frac{|v|^{q}}{|x|^{s}} d x \\
& \leq\left(\frac{N \omega_{N} R_{0}^{N-s}}{N-s}\right)^{\frac{p^{*}(s)-q}{p^{*}(s)}} A_{\mu, s}^{-\frac{q}{p}}\left(\lambda^{\frac{p}{p-q}}+\theta^{\frac{p}{p-q}}\right)^{\frac{p-q}{p}}\|(u, v)\| \|^{q}
\end{aligned}
$$


Thus

$$
\|(u, v)\| \leq\left(\frac{p^{*}(t)-q}{p^{*}(t)-p}\right)^{\frac{1}{p-p}}\left(\frac{N \omega_{N} R_{0}^{N-s}}{N-s}\right)^{\frac{p^{*}(s)-q}{p^{*}(s)(p-p)}} A_{\mu, s}^{-\frac{q}{p(p-p)}}\left(\lambda^{\frac{p}{p-q}}+\theta^{\frac{p}{p-q}}\right)^{\frac{1}{p}} .
$$

This implies

$$
\lambda^{\frac{p}{p-q}}+\theta^{\frac{p}{p-q}} \geq C
$$

This is a contradiction! Here

$$
C=\left(\frac{p-q}{p\left(p^{*}(t)-q\right)}\right)^{\frac{p}{p^{*}(t)-p}}\left(\frac{p^{*}(t)-p}{p^{*}(t)-q}\right)^{\frac{p}{p-q}}\left(\frac{N \omega_{N} R_{0}^{N-s}}{N-s}\right)^{-\frac{p\left(p^{*}(s)-q\right)}{p^{*}(s)(p-q)}} A_{\mu, s}^{\frac{q}{p-q}} A_{\mu, t}^{\frac{p^{*}(t)}{p^{*}(t)-p}}
$$

Lemma 2.2 The energy functional $J_{\lambda, \theta}$ is coercive and bounded below on $N_{\lambda, \theta}$.

Proof If $(u, v) \in N_{\lambda, \theta}$, then by (6),

$$
\begin{aligned}
J_{\lambda, \theta}(u, v)= & \frac{1}{p}\|(u, v)\|^{p}-\frac{p}{\alpha+\beta} \int_{\Omega} \frac{|u|^{\alpha}|v|^{\beta}}{|x|^{t}} d x-\frac{1}{q} K_{\lambda, \theta}(u, v) \\
\geq & \frac{p^{*}(t)-p}{p p^{*}(t)}\|(u, v)\|^{p} \\
& -\left(\frac{p^{*}(t)-q}{p^{*}(t) q}\right)\left(\frac{N \omega_{N} R_{0}^{N-s}}{N-s}\right)^{\frac{p^{*}(s)-q}{p^{*}(s)}}\left(\lambda^{\frac{p}{p-q}}+\theta^{\frac{p}{p-q}}\right)^{\frac{p-q}{p}} A_{\mu, s}^{-\frac{q}{p}}\|(u, v)\|^{q} .
\end{aligned}
$$

Since $0 \leq s, t<N, 1<q<p<p^{*}(t)$, we see that $J_{\lambda, \theta}$ is coercive and bounded below on $N_{\lambda, \theta}$.

Furthermore, similar to the argument in Brown and Zhang [[12], Theorem 2.3] (or see Binding, Drábek, and Huang [4]), we can conclude the following result. We have

Lemma 2.3 Assume that $\left(u_{0}, v_{0}\right)$ is a local minimizer for $J_{\lambda, \theta}$ on $N_{\lambda, \theta}$ and that $\left(u_{0}, v_{0}\right) \notin N_{\lambda, \theta}^{0}$. Then $J_{\lambda, \theta}^{\prime}\left(u_{0}, v_{0}\right)=0$ in $W^{-1}$.

Now, by Lemma 2.1, we let

$$
\Theta_{C_{0}}=\left\{(\lambda, \theta) \in \mathbb{R}^{2} \backslash\{(0,0)\}: 0<\lambda^{\frac{p}{p-q}}+\theta^{\frac{p}{p-q}}<C\right\},
$$

where $C_{0}=\left(\frac{q}{p}\right)^{\frac{p}{p-q}} C<C$. If $(\lambda, \theta) \in \Theta_{C_{0}}$, we have $N_{\lambda, \theta}=N_{\lambda, \theta}^{+} \cup N_{\lambda, \theta}^{-}$. Define

$$
\begin{aligned}
& \xi_{\lambda, \theta}=\inf _{(u, v) \in N_{\lambda, \theta}} J_{\lambda, \theta}(u, v) \\
& \xi_{\lambda, \theta}^{+}=\inf _{(u, v) \in N_{\lambda, \theta}^{+}} J_{\lambda, \theta}(u, v) \\
& \xi_{\lambda, \theta}^{-}=\inf _{(u, v) \in N_{\lambda, \theta}^{-}} J_{\lambda, \theta}(u, v)
\end{aligned}
$$

Lemma 2.4 There exists a positive number $C_{0}$ such that if $(\lambda, \theta) \in \Theta_{C_{0}}$, then

(i) $\xi_{\lambda, \theta} \leq \xi_{\lambda, \theta}^{+}<0$;

(ii) there exists $d_{0}=d_{0}(p, q, N, K, S, \lambda, \theta)>0$ such that $\xi_{\lambda, \theta}^{-}>d_{0}$. 
Proof (i) For $(u, v) \in N_{\lambda, \theta}^{+}$, by (10), we have

$$
K_{\lambda, \theta}(u, v) \geq \frac{p^{*}(t)-p}{p^{*}(t)-q}\|(u, v)\|^{p}
$$

and so

$$
\begin{aligned}
J_{\lambda, \theta}(u, v) & =\left(\frac{1}{p}-\frac{1}{p^{*}(t)}\right)\|(u, v)\|^{p}-\left(\frac{1}{q}-\frac{1}{p^{*}(t)}\right) K_{\lambda, \theta}(u, v) \\
& \leq\left(\frac{1}{p}-\frac{1}{p^{*}(t)}\right)\|(u, v)\|^{p}-\left(\frac{1}{q}-\frac{1}{p^{*}(t)}\right) \frac{p^{*}(t)-p}{p^{*}(t)-q}\|(u, v)\|^{p} \\
& =\frac{p^{*}(t)-p}{p^{*}(t)}\left(\frac{1}{p}-\frac{1}{q}\right)\|(u, v)\|^{p}<0 .
\end{aligned}
$$

Thus, from the definition of $\xi_{\lambda, \theta}$ and $\xi_{\lambda, \theta}^{+}$, we can deduce that $\xi_{\lambda, \theta}<\xi_{\lambda, \theta}^{+}<0$.

(ii) For $(u, v) \in N_{\lambda, \theta}^{-}$, by Lemma 2.1,

$$
\|(u, v)\| \geq\left(\frac{p-q}{p\left(p^{*}(t)-q\right)} A_{\mu, t}^{\frac{p^{*}(t)}{p}}\right)^{\frac{1}{p^{*}(t)-p}} .
$$

Moreover, by Lemma 2.2,

$$
\begin{aligned}
J_{\lambda, \theta}(u, v) \geq & \frac{p^{*}(t)-p}{p p^{*}(t)}\|(u, v)\|^{p} \\
& -\left(\frac{p^{*}(t)-q}{p^{*}(t) q}\right)\left(\frac{N \omega_{N} R_{0}^{N-s}}{N-s}\right)^{\frac{p^{*}(s)-q}{p^{*}(s)}}\left(\lambda^{\frac{p}{p-q}}+\theta^{\frac{p}{p-q}}\right)^{\frac{p-q}{p}} A_{\mu, s}^{-\frac{q}{p}}\|(u, v)\|^{q} \\
= & \|(u, v)\|^{q}\left[\frac{p^{*}(t)-p}{p p^{*}(t)}\|(u, v)\|^{p-q}\right. \\
& \left.-\left(\frac{p^{*}(t)-q}{p^{*}(t) q}\right)\left(\frac{N \omega_{N} R_{0}^{N-s}}{N-s}\right)^{\frac{p^{*}(s)-q}{p^{*}(s)}} A_{\mu, s}^{-\frac{q}{p}}\left(\lambda^{\frac{p}{p-q}}+\theta^{\frac{p}{p-q}}\right)^{\frac{p-q}{p}}\right] \\
\geq & \left(\frac{p-q}{p\left(p^{*}(t)-q\right)} A_{\mu, t}^{\frac{p^{*}(t)}{p}}\right)^{\frac{q}{p^{*}(t)-p}}\left[\frac{p^{*}(t)-p}{p p^{*}(t)}\|(u, v)\|^{p-q}\right. \\
& \left.-\left(\frac{p^{*}(t)-q}{p^{*}(t) q}\right)\left(\frac{N \omega_{N} R_{0}^{N-s}}{N-s}\right)^{\frac{p^{*}(s)-q}{p^{*}(s)}} A_{\mu, s}^{-\frac{q}{p}}\left(\lambda^{\frac{p}{p-q}}+\theta^{\frac{p}{p-q}}\right)^{\frac{p-q}{p}}\right] .
\end{aligned}
$$

Thus, if

$$
0<\lambda^{\frac{p}{p-q}}+\theta^{\frac{p}{p-q}}<C_{0}
$$

then for each $(u, v) \in N_{\lambda, \theta}^{-}$we have

$$
J_{\lambda, \theta}(u, v) \geq d_{0}=d_{0}(p, q, N, K, S, \lambda, \theta)>0 .
$$


For each $(u, v) \in W \backslash\{(0,0)\}$ such that $\int_{\Omega} \frac{|u|^{\alpha}|v|^{\beta}}{|x|^{t}} d x>0$, let

$$
t_{\max }=\left(\frac{(p-q) \|(u, v)||^{p}}{p\left(p^{*}(t)-q\right) \int_{\Omega} \frac{|u|^{\alpha}|v|^{\beta}}{|x|^{t}} d x}\right)^{\frac{1}{p^{*}(t)-p}} .
$$

Lemma 2.5 Assume that

$$
0<\lambda^{\frac{p}{p-q}}+\theta^{\frac{p}{p-q}}<C_{0}
$$

Then, for every $(u, v) \in W$ with $\int_{\Omega} \frac{|u|^{\alpha}|v|^{\beta}}{|x|^{t}} d x>0$ there exists $t_{\max }>0$ such that there are unique $t^{+}$and $t^{-}$ with $0<t^{+}<t_{\max }<t^{-}$such that $\left(t^{ \pm} u, t^{ \pm} v\right) \in N_{\lambda, \theta}^{ \pm}$and

$$
\begin{aligned}
& J_{\lambda, \theta}\left(t^{+} u, t^{+} v\right)=\inf _{0 \leq t \leq t_{\max }} J_{\lambda, \theta}(t u, t v), \\
& J_{\lambda, \theta}\left(t^{-} u, t^{-} v\right)=\sup _{t \geq t_{\max }} J_{\lambda, \theta}(t u, t v) .
\end{aligned}
$$

Proof The proof is similar to [8, Lemma 2.6] and is omitted.

Remark 2.6 If

$$
0<\lambda^{\frac{p}{p-q}}+\theta^{\frac{p}{p-q}}<C_{0} .
$$

Then, by Lemma 2.4 and Lemma 2.5 for every $(u, v) \in W$ with $\int_{\Omega} \frac{|u|^{\alpha}|v|^{\beta}}{|x|^{t}} d x>0$, we can easily deduce that there exists $t_{\max }>0$ such that there are unique $t^{-}$with $t_{\max }<t^{-}$such that $\left(t^{-} u, t^{-} v\right) \in N_{\lambda, \theta}^{-}$and

$$
J_{\lambda, \theta}\left(t^{-} u, t^{-} v\right)=\sup _{t \geq 0} J_{\lambda, \theta}(t u, t v) \geq \xi_{\lambda, \theta}^{-}>0
$$

\section{Main results}

We are now ready to state our main results.

Theorem 3.1 Assume that $0 \leq s, t<p, N \geq 3,0 \leq \mu<\bar{\mu}$ and $1 \leq q<p$. Then we have the following results:

(i) If $\lambda, \theta>0$ satisfy $\lambda^{\frac{p}{p-q}}+\theta^{\frac{p}{p-q}}<C$, then (1) has at least one positive solution in $W$.

(ii) If $\lambda, \theta>0$ satisfy $0<\lambda^{\frac{p}{p-q}}+\theta^{\frac{p}{p-q}}<C_{0}$, then (1) has at least two positive solutions in $W$.

First, we get the following result:

Lemma 3.2 (i) If $0<\lambda^{\frac{p}{p-q}}+\theta^{\frac{p}{p-q}}<C$, then there exists a $(P S)_{\xi_{\lambda, \theta}}$-sequence $\left\{\left(u_{n}, v_{n}\right)\right\} \subset N_{\lambda, \theta}$ in $W$ for $J_{\lambda, \theta}$;

(ii) If $0<\lambda^{\frac{p}{p-q}}+\theta^{\frac{p}{p-q}}<C_{0}$, then there exists a $(P S)_{\xi_{\lambda, \theta}^{-}}$-sequence $\left\{\left(u_{n}, v_{n}\right)\right\} \subset N_{\lambda, \theta}^{-}$in $W$ for $J_{\lambda, \theta}$, where $C$ is the positive constant given in Lemma 2.1, and $C_{0}=\left(\frac{q}{p}\right)^{\frac{p}{p-q}} C$.

Proof The proof is similar to [10, Proposition 9] and is omitted.

Theorem 3.3 Assume that $0 \leq s, t<p, N \geq 3,0 \leq \mu<\bar{\mu}$ and $1 \leq q<p$. If $0<\lambda^{\frac{p}{p-q}}+\theta^{\frac{p}{p-q}}<C$. Then there exists $\left(u_{0}^{+}, v_{0}^{+}\right) \in N_{\lambda, \theta}^{+}$such that

(i) $J_{\lambda, \theta}\left(u_{0}^{+}, v_{0}^{+}\right)=\xi_{\lambda, \theta}=\xi_{\lambda, \theta}^{+}$. 
(ii) $\left(u_{0}^{+}, v_{0}^{+}\right)$is a positive solution of (1),

(iii) $J_{\lambda, \theta}\left(u_{0}^{+}, v_{0}^{+}\right) \rightarrow 0$ as $\lambda \rightarrow 0^{+}, \theta \rightarrow 0^{+}$.

Proof By the Lemma 3.2, there exists a minimizing sequence $\left\{\left(u_{n}, v_{n}\right)\right\}$ for $J_{\lambda, \theta}$ on $N_{\lambda, \theta}$ such that

$$
J_{\lambda, \theta}\left(u_{n}, v_{n}\right)=\xi_{\lambda, \theta}+o(1) \text { and } J_{\lambda, \theta}^{\prime}\left(u_{n}, v_{n}\right)=o(1) \text { in } W^{-1} .
$$

Since $J_{\lambda, \theta}$ is coercive on $N_{\lambda, \theta}$ (see Lemma 2.2, ), we get $\left\{\left(u_{n}, v_{n}\right)\right\}$ is bounded in $W$. Thus, there is a subsequence $\left\{\left(u_{n}, v_{n}\right)\right\}$ and $\left.\left(u_{0}^{+}, v_{0}^{+}\right)\right) \in W$ such that

$$
\begin{cases}u_{n} \rightarrow u_{0}^{+}, v_{n} \rightarrow v_{0}^{+}, & \text {weakly in } D_{0}^{1, p}(\Omega), \\ u_{n} \rightarrow u_{0}^{+}, v_{n} \rightarrow v_{0}^{+}, & \text {weakly in } L^{p^{*}(t)}\left(\Omega,|x|^{-t}\right), \\ u_{n} \rightarrow u_{0}^{+}, v_{n} \rightarrow v_{0}^{+}, & \text {strongly in } L^{q}\left(\Omega,|x|^{-s}\right), \text { for } 1 \leq q<p^{*}(s), \\ u_{n} \rightarrow u_{0}^{+}, v_{n} \rightarrow v_{0}^{+}, & \text {a.e. in } \Omega .\end{cases}
$$

This implies that

$$
K_{\lambda, \theta}\left(u_{n}, v_{n}\right) \rightarrow K_{\lambda, \theta}\left(u_{0}^{+}, v_{0}^{+}\right), \quad \text { as } n \rightarrow \infty .
$$

By (11) and (12), it is easy to prove that $\left(u_{0}^{+}, v_{0}^{+}\right)$is a weak solution of Problem (1). Since

$$
\begin{aligned}
J_{\lambda, \theta}\left(u_{n}, v_{n}\right) & =\frac{p^{*}(t)-p}{p p^{*}(t)}\left\|\left(u_{n}, v_{n}\right)\right\|^{p}-\frac{p^{*}(t)-q}{q p^{*}(t)} K_{\lambda, \theta}\left(u_{n}, v_{n}\right) \\
& \geq-\frac{p^{*}(t)-q}{q p^{*}(t)} K_{\lambda, \theta}\left(u_{n}, v_{n}\right)
\end{aligned}
$$

and by Lemma 2.4(i),

$$
J_{\lambda, \theta}\left(u_{n}, v_{n}\right) \rightarrow \xi_{\lambda, \theta}<0 \quad \text { as } n \rightarrow \infty .
$$

Letting $n \rightarrow \infty$, we see that $K_{\lambda, \theta}\left(u_{0}^{+}, v_{0}^{+}\right)>0$.

Now, we prove that $u_{n} \rightarrow u_{0}^{+}, v_{n} \rightarrow v_{0}^{+}$strongly in $D_{0}^{1, p}(\Omega)$ and $J_{\lambda, \theta}\left(u_{0}^{+}, v_{0}^{+}\right)=\xi_{\lambda, \theta}$.

By applying Fatou's lemma and $\left(u_{0}^{+}, v_{0}^{+}\right) \in N_{\lambda, \theta}$, we get

$$
\begin{aligned}
\xi_{\lambda, \theta} & \leq J_{\lambda, \theta}\left(u_{0}^{+}, v_{0}^{+}\right)=\frac{p^{*}(t)-p}{p^{*}(t) p}\left\|\left(u_{0}^{+}, v_{0}^{+}\right)\right\|^{p}-\frac{p^{*}(t)-q}{q p^{*}(t)} K_{\lambda, \theta}\left(u_{0}^{+}, v_{0}^{+}\right) \\
& \leq \liminf _{n \rightarrow \infty}\left(\frac{p^{*}(t)-p}{p^{*}(t) p}\left\|\left(u_{n}, v_{n}\right)\right\|^{p}-\frac{p^{*}(t)-q}{q p^{*}(t)} K_{\lambda, \theta}\left(u_{n}, v_{n}\right)\right) \\
& \leq \liminf _{n \rightarrow \infty} J_{\lambda, \theta}\left(u_{n}, v_{n}\right)=\xi_{\lambda, \theta} .
\end{aligned}
$$

This implies that

$$
\begin{array}{r}
J_{\lambda, \theta}\left(u_{0}^{+}, v_{0}^{+}\right)=\xi_{\lambda, \theta}, \\
\lim _{n \rightarrow \infty}\left\|\left(u_{n}, v_{n}\right)\right\|^{p}=\left.\left\|\left(u_{0}^{+}, v_{0}^{+}\right)\right\|\right|^{p} .
\end{array}
$$

Then, $u_{n} \rightarrow u_{0}^{+}$and $v_{n} \rightarrow v_{0}^{+}$strongly in $D_{0}^{1, p}(\Omega)$.

Moreover, we have $\left(u_{0}^{+}, v_{0}^{+}\right) \in N_{\lambda, \theta}^{+}$. In fact, if $\left(u_{0}^{+}, v_{0}^{+}\right) \in N_{\lambda, \theta}^{-}$, by Lemma 2.5, there are unique $t_{0}^{+}$and $t_{0}^{-}$ such that $\left(t_{0}^{+} u_{0}^{+}, t_{0}^{+} v_{0}^{+}\right) \in N_{\lambda, \theta}^{+},\left(t_{0}^{-} u_{0}^{+}, t_{0}^{-} v_{0}^{+}\right) \in N_{\lambda, \theta}^{-}$and $t_{0}^{+}<t_{0}^{-}=1$. Since

$$
\frac{d}{d t} J_{\lambda, \theta}\left(t_{0}^{+} u_{0}^{+}, t_{0}^{+} v_{0}^{+}\right)=0 \text { and } \frac{d^{2}}{d t^{2}} J_{\lambda, \theta}\left(t_{0}^{+} u_{0}^{+}, t_{0}^{+} v_{0}^{+}\right)>0
$$


there exist $t_{0}^{+}<\bar{t} \leq t_{0}^{-}$such that $J_{\lambda, \theta}\left(t_{0}^{+} u_{0}^{+}, t_{0}^{+} v_{0}^{+}\right)<J_{\lambda, \theta}\left(\bar{t}_{0} u_{0}^{+}, \bar{t}_{0} v_{0}^{+}\right)$. By Lemma 2.5, we have

$$
\begin{aligned}
J_{\lambda, \theta}\left(t_{0}^{+} u_{0}^{+}, t_{0}^{+} v_{0}^{+}\right) & <J_{\lambda, \theta}\left(\bar{t}_{0} u_{0}^{+}, \bar{t}_{0} v_{0}^{+}\right) \\
& \leq J_{\lambda, \theta}\left(t_{0}^{-} u_{0}^{+}, t_{0}^{-} u_{0}^{+}\right) \\
& =J_{\lambda, \theta}\left(u_{0}^{+}, v_{0}^{+}\right)
\end{aligned}
$$

which contradicts $J_{\lambda, \theta}\left(u_{0}^{+}, v_{0}^{+}\right)=\xi_{\lambda, \theta}^{+}$.

Since $J_{\lambda, \theta}\left(u_{0}^{+}, v_{0}^{+}\right)=J_{\lambda, \theta}\left(\left|u_{0}^{+}\right|,\left|v_{0}^{+}\right|\right)$and $\left(\left|u_{0}^{+}\right|,\left|v_{0}^{+}\right|\right) \in N_{\lambda, \theta}^{+}$, by Lemma 2.3, we may assume that $\left(u_{0}^{+}, v_{0}^{+}\right)$ is non-negative solution of Problem (1).

Moreover, by Lemmas 2.2 and 2.4, we have

$$
\begin{aligned}
0>\xi_{\lambda, \theta} & =J_{\lambda, \theta}\left(u_{0}^{+}, v_{0}^{+}\right) \\
& \geq-\left(\frac{p^{*}(t)-q}{p^{*}(t) q}\right)\left(\frac{N \omega_{N} R_{0}^{N-s}}{N-s}\right)^{\frac{p^{*}(s)-q}{p^{*}(s)}}\left(\lambda^{\frac{p}{p-q}}+\theta^{\frac{p}{p-q}}\right)^{\frac{p-q}{p}} A_{\mu, s}^{-\frac{q}{p}}\left\|\left(u_{0}^{+}, v_{0}^{+}\right)\right\|^{q} .
\end{aligned}
$$

This implies that $J_{\lambda, \theta}\left(u_{0}^{+}, v_{0}^{+}\right) \rightarrow 0$ as $\lambda \rightarrow 0^{+}, \theta \rightarrow 0^{+}$.

Lemma 3.4 Assume that $0 \leq s, t<p, 1 \leq q<p, 0 \leq \mu<\bar{\mu}, \alpha, \beta>1$ and $\alpha+\beta=p^{*}(t)$. If

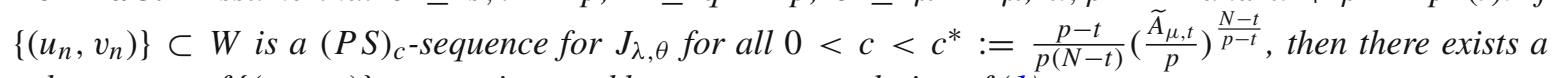
subsequence of $\left\{\left(u_{n}, v_{n}\right)\right\}$ converging weakly to a nonzero solution of $(1)$.

Proof Suppose $\left\{\left(u_{n}, v_{n}\right)\right\} \subset W$ satisfies $J_{\lambda, \theta}\left(u_{n}, v_{n}\right) \rightarrow c$ and $J_{\lambda, \theta}^{\prime}\left(u_{n}, v_{n}\right) \rightarrow 0$ with $c<c^{*}$. Since $\left\{\left(u_{n}, v_{n}\right)\right\}$ is bounded in $W$ and there exists $(u, v)$ such that $\left(u_{n}, v_{n}\right) \rightarrow(u, v)$ up to a subsequence. Moreover, we may assume

$$
\begin{cases}u_{n} \rightarrow u, \quad v_{n} \rightarrow v, & \text { weakly in } D_{0}^{1, p}(\Omega), \\ u_{n} \rightarrow u, \quad v_{n} \rightarrow v, & \text { weakly in } L^{q}\left(\Omega,|x|^{-s}\right) \text { for all } 1 \leq q<p, \\ u_{n} \rightarrow u, \quad v_{n} \rightarrow v, & \text { weakly in } L^{p^{*}(t)}\left(\Omega,|x|^{-t}\right), \\ u_{n} \rightarrow u, \quad u_{n} \rightarrow u, & \text { a.e. on } \Omega .\end{cases}
$$

Hence, we have $J_{\lambda, \theta}^{\prime}(u, v)=0$ by the weak continuity of $J_{\lambda, \theta}$. Let $\widetilde{u}_{n}=u_{n}-u, \widetilde{v}_{n}=v_{n}-v$. Then we have

$$
\left\|\left(\widetilde{u}_{n}, \widetilde{v}_{n}\right)\right\|^{p}=\left\|\left(u_{n}, v_{n}\right)\right\|^{p}-\|(u, v)\|^{p}=o(1),
$$

and by Brèzis-Lieb lemma [6], we obtain

$$
\int_{\Omega} \frac{\left|\widetilde{u}_{n}\right|^{\alpha}\left|\widetilde{v}_{n}\right|^{\beta}}{|x|^{t}} d x=\int_{\Omega} \frac{\left|u_{n}\right|^{\alpha}\left|v_{n}\right|^{\beta}}{|x|^{t}} d x-\int_{\Omega} \frac{|u|^{\alpha}|v|^{\alpha}}{|x|^{t}} d x+o(1),
$$

Hence, as $n \rightarrow \infty$,

$$
\left\langle J_{\lambda, \theta}^{\prime}\left(\widetilde{u}_{n}, \widetilde{v}_{n}\right),\left(\widetilde{u}_{n}, \widetilde{v}_{n}\right)\right\rangle=\left\langle J_{\lambda, \theta}^{\prime}\left(u_{n}, v_{n}\right),\left(u_{n}, v_{n}\right)\right\rangle-\left\langle J_{\lambda, \theta}^{\prime}(u, v),(u, v)\right\rangle+o(1)=o(1) .
$$

Consequently,

$$
\left\|\left(\widetilde{u}_{n}, \widetilde{v}_{n}\right)\right\|^{p} \rightarrow l, \quad p \int_{\Omega} \frac{\left|\widetilde{u}_{n}\right|^{\alpha}\left|\widetilde{v}_{n}\right|^{\beta}}{|x|^{t}} d x \rightarrow l,
$$

From definition of $\widetilde{A}_{\mu, t}$ and (15), we obtain

$$
\begin{aligned}
\widetilde{A}_{\mu, t}\left(\frac{l}{p}\right)^{\frac{p}{p^{*}(t)}} & =\widetilde{A}_{\mu, t} \lim _{n \rightarrow \infty}\left(\int_{\Omega} \frac{\left|\widetilde{u}_{n}\right|^{\alpha}\left|\widetilde{v}_{n}\right|^{\beta}}{|x|^{t}} d x\right)^{\frac{p}{p^{*}(t)}} \\
& \leq \lim _{n \rightarrow \infty}\left\|\left(\widetilde{u}_{n}, \widetilde{v}_{n}\right)\right\|^{p}=l
\end{aligned}
$$


which implies that either

$$
l=0 \quad \text { or } \quad l \geq p\left(\frac{\widetilde{A}_{\mu, t}}{p}\right)^{\frac{p^{*}(t)}{p^{*}(t)-p}}=p\left(\frac{\widetilde{A}_{\mu, t}}{p}\right)^{\frac{N-t}{p-t}} .
$$

Note that $\left\langle J_{\lambda, \theta}^{\prime}(u, v),(u, v)\right\rangle=0$ and

$$
J_{\lambda, \theta}(u, v)=J(u, v)-\frac{1}{p}\left\langle J^{\prime}(u, v),(u, v)\right\rangle \geq 0 .
$$

From (15) and (17), we get

$$
\begin{aligned}
c & =J_{\lambda, \theta}\left(u_{n}, v_{n}\right)+o(1) \\
& =J_{\lambda, \theta}\left(\widetilde{u}_{n}, \widetilde{v}_{n}\right)+J_{\lambda, \theta}(u, v)+o(1) \\
& \geq \frac{1}{p}\left\|\left(\widetilde{u}_{n}, \widetilde{v}_{n}\right)\right\|^{p}-\frac{1}{p^{*}(t)} \int_{\Omega} \frac{\left|\widetilde{u}_{n}\right|^{\alpha}\left|\widetilde{v}_{n}\right|^{\beta}}{|x|^{t}} d x \\
& =\frac{p^{*}(t)-p}{p p^{*}(t)} l+o(1) \\
& =\frac{p-t}{p(N-t)} l+o(1) .
\end{aligned}
$$

By (16)-(18) and the assumption $c<c^{*}$ we deduce that $l=0$. Up to a subsequence, $\left(u_{n}, v_{n}\right) \rightarrow(u, v)$ strongly in $W$.

Lemma 3.5 (see [26]) Assume that $1<p<N, 0 \leq t<p$ and $0 \leq \mu<\bar{\mu}$. Then the limiting problem

$$
\begin{cases}-\Delta_{p} u-\mu \frac{|u|^{p-1}}{|x|^{p}}=\frac{|u|^{p^{*}(t)-1}}{|x|^{t}}, & \text { in } \mathbb{R}^{N} \backslash\{0\}, \\ u \in W^{1, p}\left(\mathbb{R}^{N}\right), \quad u>0, & \text { in } \mathbb{R}^{N} \backslash\{0\},\end{cases}
$$

has positive radial ground states

$$
V_{\epsilon}(x) \triangleq \epsilon^{\frac{p-N}{p}} U_{p, \mu}\left(\frac{x}{\epsilon}\right)=\epsilon^{\frac{p-N}{p}} U_{p, \mu}\left(\frac{|x|}{\epsilon}\right), \quad \forall \epsilon>0
$$

that satisfy

$$
\int_{\Omega}\left(\left|\nabla V_{\epsilon}(x)\right|^{p}-\mu \frac{\left|V_{\epsilon}(x)\right|^{p}}{|x|^{p}}\right) d x=\int_{\Omega} \frac{\left|V_{\epsilon}(x)\right|^{p^{*}(t)}}{|x|^{t}} d x=\left(A_{\mu, t}\right)^{\frac{N-t}{p-t}},
$$

where $U_{p, \mu}(x)=U_{p, \mu}(|x|)$ is the unique radial solution of the limiting problem with

$$
U_{p, \mu}(1)=\left(\frac{(N-t)(\bar{\mu}-\mu)}{N-p}\right)^{\frac{1}{p^{*}(t)-p}} .
$$

Furthermore, $U_{p, \mu}$ have the following properties:

$$
\begin{aligned}
& \lim _{r \rightarrow 0} r^{a(\mu)} U_{p, \mu}(r)=C_{1}>0, \\
& \lim _{r \rightarrow+\infty} r^{b(\mu)} U_{p, \mu}(r)=C_{2}>0, \\
& \lim _{r \rightarrow 0} r^{a(\mu)+1}\left|U_{p, \mu}^{\prime}(r)\right|=C_{1} a(\mu) \geq 0, \\
& \lim _{r \rightarrow+\infty} r^{b(\mu)+1}\left|U_{p, \mu}^{\prime}(r)\right|=C_{2} b(\mu)>0,
\end{aligned}
$$


where $C_{i}(i=1,2)$ are positive constants and $a(\mu)$ and $b(\mu)$ are zeros of the function

$$
f(\zeta)=(p-1) \zeta^{p}-(N-p) \zeta^{p-1}+\mu, \quad \zeta \geq 0, \quad 0 \leq \mu<\bar{\mu}
$$

that satisfy

$$
0 \leq a(\mu)<\frac{N-p}{p}<b(\mu) \leq \frac{N-p}{p-1} .
$$

Now, we will give some estimates on the extremal function $V_{\epsilon}(x)$ defined in (19). For $m \in \mathbb{N}$ large, choose $\varphi(x) \in C_{0}^{\infty}\left(\mathbb{R}^{N}\right), 0 \leq \varphi(x) \leq 1, \varphi(x)=1$ for $|x| \leq \frac{1}{2 m}, \varphi(x)=0$ for $|x| \geq \frac{1}{m},\|\nabla \varphi(x)\|_{L^{p}(\Omega)} \leq 4 m$, set $u_{\epsilon}(x)=\varphi(x) V_{\epsilon}(x)$. For $\epsilon \rightarrow 0$, the behavior of $u_{\epsilon}$ has to be the same as that of $V_{\epsilon}$, but we need precise estimates of the error terms. For $1<p<N, 0 \leq s, t<p$ and $1<q<p^{*}(s)$, we have the following estimates [26]:

$$
\begin{aligned}
\int_{\Omega}\left(\left|\nabla u_{\epsilon}\right|^{p}-\mu \frac{\left|u_{\epsilon}\right|^{p}}{|x|^{p}}\right) d x & =\left(A_{\mu, t}\right)^{\frac{N-t}{p-t}}+O\left(\epsilon^{b(\mu) p+p-N}\right), \\
\int_{\Omega} \frac{\left|u_{\epsilon}\right|^{p^{*}(t)}}{|x|^{t}} d x & =\left(A_{\mu, t}\right)^{\frac{N-t}{p-t}}+O\left(\epsilon^{b(\mu) p^{*}(t)-N+t}\right), \\
\int_{\Omega} \frac{\left|u_{\epsilon}\right|^{q}}{|x|^{s}} d x & \geq \begin{cases}C \epsilon^{N-s+\left(1-\frac{N}{p}\right) q}, & q>\frac{N-s}{b(\mu)}, \\
C \epsilon^{N-s+\left(1-\frac{N}{p}\right) q}|\ln \epsilon|, & q=\frac{N-s}{b(\mu)}, \\
C \epsilon^{q\left(b(\mu)+1-\frac{N}{p}\right) q}, & q<\frac{N-s}{b(\mu)} .\end{cases}
\end{aligned}
$$

Lemma 3.6 Assume that $0 \leq s, t<p, 1 \leq q<p, 0 \leq \mu<\bar{\mu}, \alpha, \beta>1$ and $\alpha+\beta=p^{*}(t)$. There exists a non-negative function $(u, v) \in W \backslash\{(0,0)\}$ and $\delta_{1}>0$ such that for $\lambda, \theta>0$ satisfy $0<\lambda^{\frac{p}{p-q}}+\theta^{\frac{p}{p-q}}<\delta_{1}$, we have

$$
\sup _{\tau \geq 0} J(\tau u, \tau v)<c^{*}:=\frac{p-t}{p(N-t)}\left(\frac{\widetilde{A}_{\mu, t}}{p}\right)^{\frac{N-t}{p-t}}
$$

In particular, $\xi_{\lambda, \theta}<\frac{p-t}{p(N-t)}\left(\frac{\widetilde{A}_{\mu, t}}{p}\right)^{\frac{N-t}{p-t}}$ for all $0<\lambda^{\frac{p}{p-q}}+\theta^{\frac{p}{p-q}}<\delta_{1}$.

Proof Set $u=\sqrt[p]{\alpha} u_{\epsilon}$ and $v=\sqrt[p]{\beta} u_{\epsilon}$. Then, we consider the functions

$$
\begin{aligned}
g(\tau)= & J_{\lambda, \theta}\left(\tau \sqrt[p]{\alpha} u_{\epsilon}, \tau \sqrt[p]{\beta} u_{\epsilon}\right)=\frac{\tau^{p}}{p}\left\|\left(\sqrt[p]{\alpha} u_{\epsilon}, \sqrt[p]{\beta} u_{\epsilon}\right)\right\|^{p}-\frac{\tau^{q}}{q} K_{\lambda, \theta}\left(\sqrt[p]{\alpha} u_{\epsilon}, \sqrt[p]{\beta} u_{\epsilon}\right) \\
& -\frac{p \tau^{p^{*}(t)}}{p^{*}(t)} \int_{\Omega} \frac{\left|p \sqrt[p]{\alpha} u_{\epsilon}\right|^{\alpha}\left|\sqrt[p]{\beta} u_{\epsilon}\right|^{\beta}}{|x|^{t}} d x \\
g_{1}(\tau)= & \frac{\tau^{p}}{p} \|\left.\left(\sqrt[p]{\alpha} u_{\epsilon}, \sqrt[p]{\beta} u_{\epsilon}\right)\right|^{p}-\frac{p \tau^{p^{*}(t)}}{p^{*}(t)} \int_{\Omega} \frac{\left|\sqrt[p]{\alpha} u_{\epsilon}\right|^{\alpha}\left|\sqrt[p]{\beta} u_{\epsilon}\right|^{\beta}}{|x|^{t}} d x .
\end{aligned}
$$

By (5), (24) and the fact that

$$
\begin{aligned}
\sup _{\tau \geq 0}\left(\frac{\tau^{p}}{p} A-\frac{p \tau^{p^{*}(t)}}{p^{*}(t)} B\right) & =\frac{p^{*}(t)-p}{p p^{*}(t)} A\left(\frac{A}{p B}\right)^{\frac{p}{p^{*}(t)-p}}=\frac{p^{*}(t)-p}{p p^{*}(t)}\left(\frac{A}{(p B)^{\frac{p}{p^{*}(t)}}}\right)^{\frac{p^{*}(t)}{p^{*}(t)-p}} \\
& =\frac{p-t}{p(N-t)}\left(\frac{A}{(p B)^{\frac{p}{p^{*}(t)}}}\right)^{\frac{N-t}{p-t}}, \quad A, B>0
\end{aligned}
$$


we conclude that

$$
\begin{aligned}
\sup _{\tau \geq 0} g_{1}(\tau) & \leq \frac{p-t}{p(N-t)}\left(\frac{(\alpha+\beta) \int_{\Omega}\left(\left|\nabla u_{\epsilon}\right|^{p}-\mu \frac{\left|u_{\epsilon}\right|^{p}}{|x|^{p}}\right) d x}{\left(p \alpha^{\frac{\alpha}{p}} \beta^{\frac{\beta}{p}} \int_{\Omega} \frac{\left|u_{\epsilon}\right|^{p^{*}(t)}}{|x|^{t}} d x\right)^{\frac{p}{p^{*}(t)}}}\right)^{\frac{N-t}{p-t}} \\
& \leq \frac{p-t}{p(N-t)}\left(\frac{1}{p}\right)^{\frac{N-t}{p-t}}\left(\left(\frac{\alpha}{\beta}\right)^{\frac{\beta}{\alpha+\beta}}+\left(\frac{\alpha}{\beta}\right)^{\frac{-\alpha}{\alpha+\beta}}\right)^{\frac{N-t}{p-t}}\left(\frac{\left(A_{\mu, t}\right)^{\frac{N-t}{p-t}}+O\left(\epsilon^{b(\mu) p+p-N}\right)}{\left(\left(A_{\mu, t}\right)^{\frac{N-t}{p-t}}+O\left(\epsilon^{\left.\left.b(\mu) p^{*}(t)-N+t\right)\right)}\right)\right)^{\frac{p}{p^{*}(t)}}}\right)^{\frac{N-t}{p-t}} \\
& \leq \frac{p-t}{p(N-t)}\left(\frac{1}{p}\right)^{\frac{N-p}{p-t}}\left(\left(\frac{\alpha}{\beta}\right)^{\frac{\beta}{\alpha+\beta}}+\left(\frac{\alpha}{\beta}\right)^{\frac{-\alpha}{\alpha+\beta}}\right)^{\frac{N-t}{p-t}}\left(A_{\mu, t}+o\left(\epsilon^{b(\mu) p+p-N}\right)\right)^{\frac{N-t}{p-t}} \\
& \leq \frac{p-t}{p(N-t)}\left(\frac{1}{p}\right)^{\frac{N-p}{p-t}}\left(\left(\frac{\alpha}{\beta}\right)^{\frac{\beta}{\alpha+\beta}}+\left(\frac{\alpha}{\beta}\right)^{\frac{-\alpha}{\alpha+\beta}}\right)^{\frac{N-t}{p-t}}\left(A_{\mu, t}^{\frac{N-t}{p-t}}+o\left(\epsilon^{b(\mu) p+p-N}\right)\right) \\
& =\frac{p-t}{p(N-t)}\left(\frac{\widetilde{A}_{\mu, t}}{p}\right)^{\frac{N-t}{p-t}}+o\left(\epsilon^{b(\mu) p+p-N}\right) .
\end{aligned}
$$

On the other hand, using the definitions of $g$ and $u_{\epsilon}$, we get

$$
g(\tau)=J_{\lambda, \theta}\left(\tau \sqrt[p]{\alpha} u_{\epsilon}, \tau \sqrt[p]{\beta} u_{\epsilon}\right) \leq \frac{t^{p}}{p}\left\|\left(\sqrt[p]{\alpha} u_{\epsilon}, \sqrt[p]{\beta} u_{\epsilon}\right)\right\|^{p}, \text { for all } \tau \geq 0 \text { and } \lambda>0, \theta>0 .
$$

combining this with (20), let $\epsilon \in(0,1)$, then there exists $\tau_{0} \in(0,1)$ independent of $\epsilon$ such that

$$
\sup _{0 \leq \tau \leq \tau_{0}} g(\tau)<\frac{p-t}{p(N-t)}\left(\frac{\widetilde{A}_{\mu, t}}{p}\right)^{\frac{N-t}{p-t}}, \quad \text { for all } 0<\lambda^{\frac{p}{p-q}}+\theta^{\frac{p}{p-q}}<\delta_{1} .
$$

Hence, as $0<\lambda^{\frac{p}{p-q}}+\theta^{\frac{p}{p-q}}<\delta_{1}, 1 \leq q<p$, by (25), we have that

$$
\begin{aligned}
\sup _{\tau \geq \tau_{0}} g(\tau)= & \sup _{\tau \geq \tau_{0}}\left(g_{1}(\tau)-\frac{\tau^{q}}{q} K_{\lambda, \theta}\left(\sqrt[p]{\alpha} u_{\epsilon}, \sqrt[p]{\beta} u_{\epsilon}\right)\right) \\
\leq & \frac{p-t}{p(N-t)}\left(\frac{\widetilde{A}_{\mu, t}}{p}\right)^{\frac{N-t}{p-t}}+o\left(\epsilon^{b(\mu) p+p-N}\right) \\
& -\frac{\tau_{0}^{q}}{q}\left(\alpha^{\frac{q}{p}} \lambda+\beta^{\frac{q}{p}} \theta\right) \int_{\Omega} \frac{\left|u_{\epsilon}\right|^{q}}{|x|^{s}} d x .
\end{aligned}
$$

(i) If $1 \leq q<\frac{N-s}{b(\mu)}$, then by (22), one can get

$$
\int_{\Omega} \frac{\left|u_{\epsilon}\right|^{q}}{|x|^{s}} d x \geq C \epsilon^{q\left(b(\mu) p+1-\frac{N}{p}\right)}
$$

and since $b(\mu)>\frac{N-p}{p}$, then

$$
(b(\mu) p+p-N)>q\left(b(\mu) p+1-\frac{N}{p}\right) .
$$

Combining this with (26) and (27), for any $\lambda, \theta>0$ which $0<\lambda^{\frac{p}{p-q}}+\theta^{\frac{p}{p-q}}<\delta_{1}$ we can choose $\epsilon$ small enough such that

$$
\sup _{\tau \geq 0} J_{\lambda, \theta}\left(\tau \sqrt[p]{\alpha} u_{\epsilon}, \tau \sqrt[p]{\beta} u_{\epsilon}\right)<\frac{p-t}{p(N-t)}\left(\frac{\widetilde{A}_{\mu, t}}{p}\right)^{\frac{N-t}{p-t}} .
$$


(ii) If $\frac{N-s}{b(\mu)} \leq q<p$, then by (22) and $b(\mu)>\frac{N-p}{p}$ we have that

$$
\int_{\Omega} \frac{\left|u_{\epsilon}\right|^{q}}{|x|^{s}} d x \geq \begin{cases}C \epsilon^{N-s+\left(1-\frac{N}{p}\right) q}, & q>\frac{N-s}{b(\mu)} \\ C \epsilon^{N-s+\left(1-\frac{N}{p}\right) q}|\ln \epsilon|, & q=\frac{N-s}{b(\mu)}\end{cases}
$$

and

$$
(b(\mu) p+p-N)>N-s+\left(1-\frac{N}{p}\right) q .
$$

Combining this with (26) and (27), for any $\lambda, \theta>0$ which $0<\lambda^{\frac{p}{p-q}}+\theta^{\frac{p}{p-q}}<\delta_{1}$ we can choose $\epsilon$ small enough such that

$$
\sup _{\tau \geq 0} J_{\lambda, \theta}\left(\tau \sqrt[p]{\alpha} u_{\epsilon}, \tau \sqrt[p]{\beta} u_{\epsilon}\right)<\frac{p-t}{p(N-t)}\left(\frac{\widetilde{A}_{\mu, t}}{p}\right)^{\frac{N-t}{p-t}} .
$$

From (i) and (ii), (23) holds.

From Lemma 2.5, (23) and the definitions of $\xi_{\lambda, \theta}^{-}$, for any $\lambda, \theta>0$ which $0<\lambda^{\frac{p}{p-q}}+\theta^{\frac{p}{p-q}}<\delta_{1}$ we obtain that there exists $\tau_{\lambda, \theta}^{-}$such that $\left(\tau_{\lambda, \theta}^{-} \sqrt[p]{\alpha} u_{\epsilon}, \tau_{\lambda, \theta}^{-} \sqrt[p]{\beta} u_{\epsilon}\right) \in N_{\lambda, \theta}^{-}$and

$$
\xi_{\lambda, \theta}^{-} \leq J_{\lambda, \theta}\left(\tau_{\lambda, \theta}^{-} \sqrt[p]{\alpha} u_{\epsilon}, \tau_{\lambda, \theta}^{-} \sqrt[p]{\beta} u_{\epsilon}\right) \leq \sup _{\tau \geq 0} J_{\lambda, \theta}\left(\tau \sqrt[p]{\alpha} u_{\epsilon}, \tau \sqrt[p]{\beta} u_{\epsilon}\right)<\frac{p-t}{p(N-t)}\left(\frac{\widetilde{A}_{\mu, t}}{p}\right)^{\frac{N-t}{p-t}} .
$$

The proof is complete.

Theorem 3.7 Assume that $0 \leq s, t<p, 1 \leq q<p, 0 \leq \mu<\bar{\mu}, \alpha, \beta>1$ and $\alpha+\beta=p^{*}(t)$. There exists $\Lambda>0$ such that for any $\lambda, \theta>0$ satisfy $0<\lambda^{\frac{p}{p-q}}+\theta^{\frac{p}{p-q}}<\Lambda$, the functional $J_{\lambda, \theta}$ has a minimizer $(U, V)$ in $N_{\lambda, \theta}^{-}$and satisfies the following:

(i) $J_{\lambda, \theta}(U, V)=\xi_{\lambda, \theta}^{-}$,

(ii) $(U, V)$ is positive solution of (1), where $\Lambda=\min \left\{C_{0}, \delta_{1}\right\}$

Proof If $0<\lambda^{\frac{p}{p-q}}+\theta^{\frac{p}{p-q}}<C_{0}=\left(\frac{q}{p}\right)^{\frac{p}{p-q}} C$, then by the Lemmas 2.4(ii), 3.2 and 3.6, there exists a $(P S)_{\xi \lambda, \theta^{-}}$ sequence $\left\{\left(u_{n}, v_{n}\right)\right\} \subset N_{\lambda, \theta}^{-}$in $W$ for $J_{\lambda, \theta}$ with $\xi_{\lambda, \theta}^{-} \in\left(0, \frac{p-t}{p(N-t)}\left(\frac{\widetilde{A}_{\mu, t}}{p}\right)^{\frac{N-t}{p-t}}\right)$. By Lemma $2.2,\left\{\left(u_{n}, v_{n}\right)\right\}$ is bounded in $W$. From Lemma 3.4, there exists a subsequence denoted by $\left\{\left(u_{n}, v_{n}\right)\right\}$ and nontrivial solution $(U, V) \in W$ of (1) such that $u_{n} \rightarrow U, v_{n} \rightarrow V$ weakly in $D_{0}^{1, p}(\Omega)$.

First, we prove that $(U, V) \in N_{\lambda, \theta}^{-}$. Arguing by contradiction, we assume $(U, V) \in N_{\lambda, \theta}^{+}$. Since $N_{\lambda, \theta}^{-}$is closed in $W_{0}^{1, p}(\Omega)$, we have $\|(U, V)\|<\liminf _{n \rightarrow \infty}\left\|\left(u_{n}, v_{n}\right)\right\|$. Thus, by Lemma 2.5 , there exists a unique $\tau^{-}$ such that $\left(\tau^{-} U, \tau^{-} V\right) \in N_{\lambda, \theta}^{-}$. If $(u, v) \in N_{\lambda, \theta}^{-}$, then it is easy to see that

$$
J_{\lambda, \theta}(u, v)=\frac{p-t}{p(N-t)}\|(u, v)\|^{p}-\frac{p^{*}(t)-q}{q p^{*}(t)} K_{\lambda, \theta}(u, v) .
$$

From Remark $2.6\left(u_{n}, v_{n}\right) \in N_{\lambda, \theta}^{-},\|(U, V)\|<\liminf _{n \rightarrow \infty}\left\|\left(u_{n}, v_{n}\right)\right\|$ and (28), we can get

$$
\xi_{\lambda, \theta}^{-} \leq J_{\lambda, \theta}\left(\tau^{-} U, \tau^{-} V\right) \leq \lim _{n \rightarrow \infty} J_{\lambda, \theta}\left(\tau^{-} u_{n}, \tau^{-} v_{n}\right)<\lim _{n \rightarrow \infty} J_{\lambda, \theta}\left(u_{n}, v_{n}\right)=\xi_{\lambda, \theta}^{-} .
$$

This is a contradiction. Thus, $(U, V) \in N_{\lambda, \theta}^{-}$, 
Next, by the same argument as that in Theorem 3.3, we get that $\left(u_{n}, v_{n}\right) \rightarrow(U, V)$ strongly in $W$ and $J_{\lambda, \theta}(U, V)=\xi_{\lambda, \theta}^{-}>0$ for all $0<\lambda^{\frac{p}{p-q}}+\theta^{\frac{p}{p-q}}<C_{0}=\left(\frac{q}{p}\right)^{\frac{p}{p-q}} C$. Since $J_{\lambda, \theta}(U, V)=J_{\lambda, \theta}(|U|,|V|)$ and $(|U|,|V|) \in N_{\lambda, \theta}^{-}$, by Lemma 2.3 we may assume that $(U, V)$ is a nontrivial nonnegative solution of (1). Finally, by the maximum principle [30], we obtain that $(U, V)$ is a positive solution of (1). The proof is complete.

Proof of Theorem 3.1 The part (i) of Theorem 3.1 immediately follows from Theorem 3.3. When $0<\lambda^{\frac{p}{p-q}}+$ $\theta^{\frac{p}{p-q}}<C_{0}=\left(\frac{q}{p}\right)^{\frac{p}{p-q}} C<C$, by Theorems 3.3 and 3.7, we obtain (1) has at least two positive solutions $\left(u_{0}, v_{0}\right)$ and $(U, V)$ such that $\left(u_{0}, v_{0}\right) \in N_{\lambda, \theta}^{+}$and $(U, V) \in N_{\lambda, \theta}^{-}$. Since $N_{\lambda, \theta}^{+} \cap N_{\lambda, \theta}^{-}=\emptyset$, this implies that $N_{\lambda, \theta}^{+}$and $N_{\lambda, \theta}^{-}$are distinct. This completes the proof of Theorem 3.1.

Acknowledgments The authors would like to thank the referees for their interesting comments and suggestions.

Open Access This article is distributed under the terms of the Creative Commons Attribution License which permits any use, distribution, and reproduction in any medium, provided the original author(s) and the source are credited.

\section{References}

1. Afrouzi, G.A.; Rasouli, S.H.: The Nehari manifold for a class of concave-convex elliptic systems involving the p-laplacian and nonlinear boundary condition. Nonlinear Anal. 73, 3390-3401 (2010)

2. Alves, C.O.; El Hamidi, A.: Nehari manifold and existence of positive solutions to a class of quasilinear problems. Nonlinear Anal. 60, 611-624 (2005)

3. Ambrosetti, A.; Rabinowitz, P.H.: Dual variational methods in critical point theory and applications. J. Funct. Anal. 14, 349-381 (1973)

4. Binding, P.A.; Drabek, P.; Huang, Y.X.: On Neumann boundary value problems for some quasilinear elliptic equations. Electron. J. Differential Equations 5, 1-11 (1997)

5. Bozhkov, Y.; Mitidieri, E.: Existence of multiple solutions for quasilinear systems via fibering method. J. Differential equations 190, 239-267 (2003)

6. Brèzis, H.; Nirenberg, L.: Positive solutions of nonlinear elliptic equations involving critical Sobolev exponents. Comm. Pure Appl. Math. 36, 437-477 (1983)

7. Brown, K.J.: The Nehari manifold for a semilinear elliptic equation involving a sublinear term. Calc. Var. 22, 483-494 (2005)

8. Brown, K.J.; Wu, T.F.: A semilinear elliptic system involving nonlinear boundary condition and sign changing weight function. J. Math. Anal. Appl. 337, 1326-1336 (2008)

9. Brown, K.J.; Wu, T.F.: A fibering map approach to a semilinear elliptic boundary value problem. Electron. J. Differential Equations 69, 1-9 (2007)

10. Brown, K.J.; Wu, T.F.: On semilinear elliptic equations involving concave-convex nonlinearities and sign-changing weight function. J. Math. Anal. Appl. 318, 253-270 (2006)

11. Brown, K.J.; Wu, T.F.: Multiplicity of positive solution of p-Laplacian problems with sign-changing weight function. Int. J. Math. Anal. 1(12), 557-563 (2007)

12. Brown, K.J.; Zhang, Y.: The Nehari manifold for a semilinear elliptic problem with a sign changing weight function. J. Differential Equations 193, 481-499 (2003)

13. Caffarelli, L.; Kohn, R.; Nirenberg, L.: First order interpolation inequality with weights. Compos. Math. 53, 259-275 (1984)

14. Cao, D.; Han, P.: Solutions for semilinear elliptic equations with critical exponents and Hardy potential. J. Differential Equations 205, 521-537 (2004)

15. Cao, D.; Kang, D.: Solutions of quasilinear elliptic problems involving a Sobolev exponent and multiple Hardy-type terms. J. Math. Anal. Appl. 333, 889-903 (2007)

16. Demengel, F.; Hebey, E.: On some nonlinear equations involving the p-Laplacian with critical Sobolev growth. Adv. Differential Equations 3, 533-574 (1998)

17. Filippucci, R.; Pucci, P.; Robert, F.: On a p-Laplace equation with multiple critical nonlinearities. J. Math. Pures Appl. 91, $156-177$ (2009)

18. Garcia Azorero, J.; Peral, I.: Hardy inequalities and some critical elliptic and parabolic problems. J. Differential Equations 144, 441-476 (1998)

19. Ghoussoub, N.; Robert, F.: The effect of curvature on the best constant in the Hardy-Sobolev inequality. Geom. Funct. Anal. 16, 897-908 (2006)

20. Ghoussoub, N.; Robert, F.: Concentration estimates for Emden-Fowler equations with boundary singularities and critical growth. Int. Math. Res. Pap. IMRP 2006, Article ID 21867, 1-85

21. Ghoussoub, N.; Yuan, C.: Multiple solutions for quasi-linear PDEs involving the critical Sobolev and Hardy exponents. Trans. Am. Math. Soc. 352, 5703-5743 (2000)

22. Han, P. Quasilinear elliptic problems with critical exponents and Hardy terms. Nonlinear Anal. 61, 735-758 (2005) 
23. Hsu, T.S.: Multiple positive solutions for a critical quasilinear elliptic system with concave-convex nonlinearities. Nonlinear Anal. 71, 2688-2698 (2009)

24. Hsu, T.S.: Multiple positive solutions for a quasilinear elliptic problem involving critical Sobolev-Hardy exponents and concave-convex nonlinearities. Nonlinear Anal. 74, 3934-3944 (2011)

25. Kang, D.: On the quasilinear elliptic problem with a critical Hardy-Sobolev exponent and a Hardy term. Nonlinear Anal. 69, 2432-2444 (2008)

26. Kang, D.: On the quasilinear elliptic problems with critical Hardy-Sobolev exponents and Hardy terms. Nonlinear Anal. 68, 1973-1985 (2008)

27. Kang, D.; Peng, S.: Solutions for semilinear elliptic problems with critical Sobolev-Hardy exponents and Hardy potential. Appl. Math. Lett. 18, 1094-1100 (2005)

28. Nehari, Z.: On a class of nonlinear second-order differential equations. Trans. Am. Math. Soc. 95, 101-123 (1960)

29. Rabinowitz, P.: Minimax methods in critical points theory with applications to differential equations. in: CBMS Regional Conference Series in Mathematics, Providence, RI, 65, 1986

30. Vazquez, J.L.: A strong maximum principle for some quasilinear elliptic equations. Appl. Math. Optim. 12, 191-202 (1984) 